\title{
Accurate mass and radius determinations of a cool subdwarf in an eclipsing binary
}

\author{
Alberto Rebassa-Mansergas ${ }^{1,2, *}$, Steven G. Parsons ${ }^{3}$, Vikram S. Dhillon ${ }^{3,4}$, Juanjuan \\ Ren $^{5}$, Stuart P. Littlefair ${ }^{3}$, Thomas R. Marsh ${ }^{6}$, and Santiago Torres ${ }^{1,2}$
}

\author{
${ }^{1}$ Departament de Física, Universitat Politècnica de Catalunya, c/Esteve Terrades 5, 08860 Castelldefels, Spain \\ ${ }^{2}$ Institut d'Estudis Espacials de Catalunya, Ed. Nexus-201, c/Gran Capità 2-4, 08034 Barcelona, Spain \\ ${ }^{3}$ Department of Physics \& Astronomy, University of Sheffield, Sheffield S3 7RH, UK \\ ${ }^{4}$ Instituto de Astrofísica de Canarias, Via Lactea s/n, La Laguna, E-38205 Tenerife, Spain \\ ${ }^{5}$ National Astronomical Observatories, Chinese Academy of Sciences, 100012 Beijing, P. R. China \\ ${ }^{6}$ Department of Physics, Gibbet Hill Road, University of Warwick, Coventry, CV4 7AL, UK \\ *alberto.rebassa@upc.edu
}

\begin{abstract}
Cool subdwarfs are metal-poor low-mass stars that formed during the early stages of the evolution of our Galaxy. Because they are relatively rare in the vicinity of the Sun, we know of few cool subdwarfs in the solar neighbourhood, and none with both the mass and the radius accurately determined. This hampers our understanding of stars at the low-mass end of the main-sequence. Here we report the discovery of SDSS J235524.29+044855.7 as an eclipsing binary containing a cool subdwarf star, with a white dwarf companion. From the light-curve and the radial-velocity curve of the binary we determine the mass and the radius of the cool subdwarf and we derive its effective temperature and luminosity by analysing its spectral energy distribution. Our results validate the theoretical mass-radius-effective temperature-luminosity relations for low-mass low-metallicity stars.
\end{abstract}

\section{Introduction}

Cool subdwarfs are metal-poor, low-mass main-sequence stars of spectral type from mid $\mathrm{K}$ to late $\mathrm{M}$. They are generally referred to as $\mathrm{sdM} / \mathrm{sdK}$ stars. The low metallicity in cool subdwarfs decreases the opacity of their outer layers resulting in a small radius as compared to a main-sequence star of the same effective temperature ${ }^{1}$. Thus, cool subdwarfs have lower luminosities than dwarfs of the same effective temperature and lie below the main sequence on the Hertzprung-Russell diagram $^{2}$. The low metallicity of cool subdwarfs implies that they are typically very old $(\gtrsim 10 \mathrm{Gyr})^{3}$ and are part of old Galactic populations such as the thick disc, the halo and the bulge ${ }^{4}$. Hence, cool subdwarfs formed in the first phases of our Galaxy and therefore carry important information regarding its structure and chemical evolution. Moreover, the analysis of the spectral energy distribution (SED) of cool subdwarfs improves our understanding of the role of metallicity in the opacity structure and evolution of cool atmospheres ${ }^{5}$. Depending on their metallicity, cool subdwarf stars are divided into three sub-classes: sd (subdwarfs), esd (extreme subdwarfs) and usd (ultra subdwarfs) ${ }^{6}$, with the metallicity content decreasing from the sd to the usd stars. Specifically, the iron abundance $[\mathrm{Fe} / \mathrm{H}]$ ranges from -0.34 to -0.87 dex for sd stars, from -0.87 to -1.36 dex for esd stars and it is lower than -1.36 dex for usd stars ${ }^{7}$. This classification is based on the strength of the TiO and $\mathrm{CaH}$ bands in the observed spectra ${ }^{8}$.

In order to understand the nature of these rare and important objects, it is essential to determine their most basic stellar parameters such as masses and radii. To that end, and despite their intrinsic low luminosity, modern large-scale surveys such as the Sloan Digital Sky Survey (SDSS) ${ }^{9}$ and the Large Sky Area Multi-Object Fiber Spectroscopic Telescope (LAMOST) ${ }^{10}$ have identified $\sim 5000$ cool subdwarfs during the last few years ${ }^{11,12}$. A recent ground-based spectroscopic survey allowed radii determinations of $88 \mathrm{cool}$ subdwarfs via the analysis of their SEDs and the use of Gaia parallaxes ${ }^{6}$. However, we currently know of only six cool subdwarfs for which dynamical masses have been measured ${ }^{14}$. Moreover, it has to be stressed that no mass and radius values for a single cool subdwarf have been measured to date. Thus, the bottom-end of the main sequence remains unconstrained for metal-poor stars.

Here we report the discovery of SDSS J235524.29+044855.7 (hereafter SDSS J2355+0448) as an eclipsing binary containing a cool subdwarf, with a white dwarf companion. SDSS J2355+0448 was originally identified as a white dwarf candidate ${ }^{15}$ and later re-discovered as part of our systematic search of white dwarf plus low-mass main sequence binaries within the SDSS and LAMOST spectroscopic data bases ${ }^{16,17}$ (see the optical spectrum in Figure 1). Eclipsing binaries offer the opportunity to 
measure directly the masses and radii of the two components with unprecedented precision ${ }^{16,19}$. This is the case for the cool subdwarf in SDSS J2355+0448.

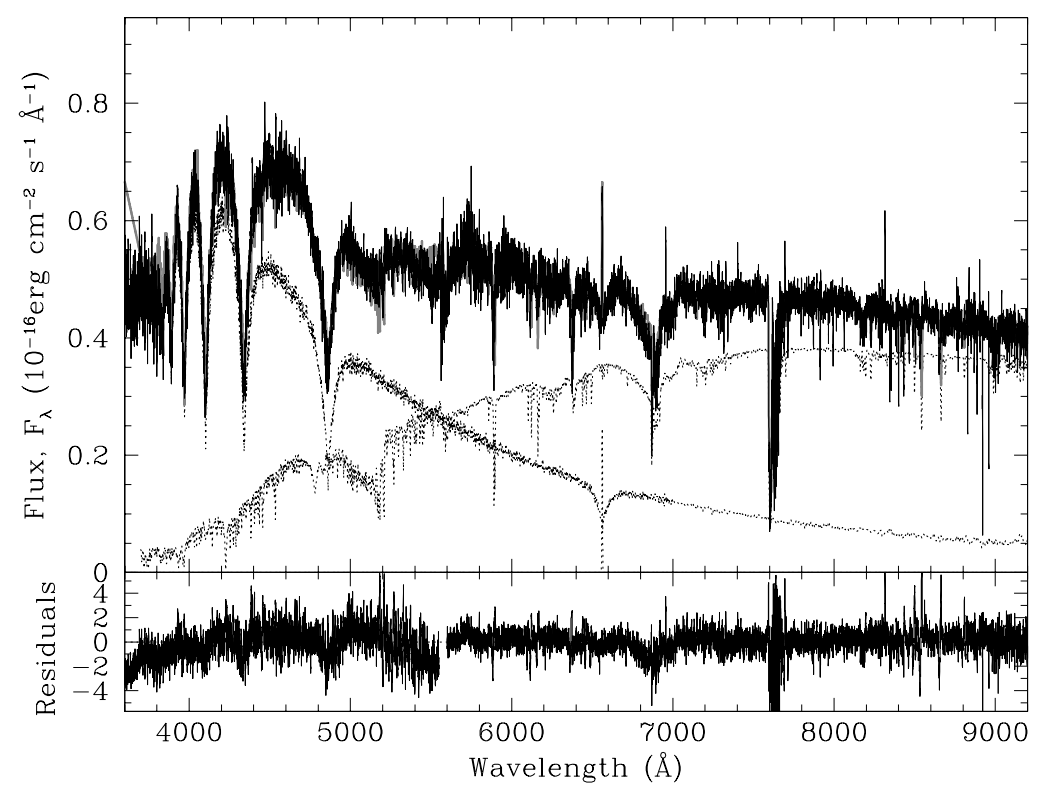

Figure 1. Top panel: X-Shooter optical spectrum of SDSS J2355+0448 (black solid lines). The hydrogen Balmer absorption lines of a DA white dwarf can be clearly seen in the blue. At longer wavelengths the spectrum is mostly featureless, with the exception of the broad $\mathrm{CaH}$ molecular band at $\sim 6800 \AA$, typical of cool subdwarfs. The strong H $\alpha$ emission arises most likely due to magnetic activity of the cool subdwarf and/or from wind accretion onto the white dwarf. The two-component template spectra (white dwarf and cool subdwarf) that best fit the observed spectrum are shown as black dotted lines. The bottom panel shows the residuals from the fit. The spectrum has been binned by a factor of five for clarity.

\section{Results}

Available photometry from the Catalina Sky Surveys ${ }^{20}$ revealed the binary star SDSS J2355+0448 to be eclipsing with an orbital period of 2.15 hours. We were awarded Director's Discretionary Time at the $8.2 \mathrm{~m}$ Very Large Telescope to obtain $\mathrm{X}$-Shooter spectra covering the entire optical plus near-infrared wavelengths of the target (see the Methods section for a description of the observations). From the spectra we derived the white dwarf effective temperature and surface gravity (hence its cooling age), the systemic velocity of the binary, the radial-velocity curve amplitude of the cool subdwarf and its metallicity class, and we estimated the cool subdwarf's $[\mathrm{Fe} / \mathrm{H}]$ abundance.

We were also awarded time on the $10.4 \mathrm{~m}$ Gran Telescopio Canarias equipped with HiPERCAM ${ }^{2}$ to sample the orbital light curves in five filters (see also the Methods section for a description of the observations). The data revealed not only the primary but also the secondary eclipse (i.e. the transit of the white dwarf in front of the cool subdwarf). The latter is crucial in fixing the orbital inclination and stellar radii relative to the orbital separation. The analysis of the HiPERCAM light-curve, together with the radial-velocity curve of the cool subdwarf, allowed precise determinations of the masses and radii of both stellar components as well as measurements of the SDSS magnitudes for the cool subdwarf. These magnitudes, together with available near-infrared photometry of the binary, permitted the effective temperature and the bolometric luminosity of the cool subdwarf to be determined via SED fitting.

The fitted and derived stellar and binary parameters of SDSS J2355+0448 are provided in Table 1.

\section{The white dwarf stellar parameters}

The optical spectrum of SDSS J2355+0448 (top panel of Figure 1) clearly shows the white dwarf and cool subdwarf components. To derive the stellar parameters of the white dwarf via Balmer-line fitting (namely the effective temperature and the surface gravity) it is necessary to subtract the cool subdwarf contribution. To that end we applied a decomposition/fitting routine $^{3}$ to the individual X-Shooter optical spectra of the binary. Note that we did not use a combined optical X-Shooter spectrum for this purpose since we were not able to measure the radial velocities of the white dwarf, and hence the Balmer lines in a combined spectrum would have suffered from orbital smearing. 
The decomposition/fitting routine first fits the two-composite spectrum with a grid of observed cool subdwarf ${ }^{5}$ and white dwarf $^{3}$ templates and subtracts the best-fitted cool subdwarf template, appropriately scaled in flux (see the best two-composite fit to one of our X-Shooter spectra in the top panel of Figure 1). In a second step, the routine fits both the normalised Balmer lines and the whole spectrum (continuum plus lines) of the residual white dwarf spectrum with a white dwarf model atmosphere grid $^{7}$ to derive the white dwarf effective temperature and surface gravity (see Supplementary Figure 1). For further details on our decomposition/fitting routine we point the reader to the Methods section.

\begin{tabular}{lccc}
\hline Parameter & units & value & uncertainty \\
\hline Orbital period & days & 0.0897780065 & 0.0000000024 \\
Binary inclination & degrees & 89.7 & 0.2 \\
Binary separation & $\mathrm{R}_{\odot}$ & 0.711 & 0.003 \\
Mass ratio & $\mathrm{M}_{\mathrm{sd}} / \mathrm{M}_{\mathrm{WD}}$ & 0.335 & 0.002 \\
Centre of WD eclipse & $\mathrm{MJD}(\mathrm{BTDB})$ & 58074.1076830 & 0.0000087 \\
Gaia parallax & $\mathrm{mas}$ & 1.89 & 0.46 \\
Bayesian distance & $\mathrm{pc}$ & 541 & $-124+216$ \\
Right ascension & $\mathrm{deg}$ & 358.85133 & \\
Declination & $\mathrm{deg}$ & 4.81539 & \\
SDSS $g$ & $\mathrm{mag}$ & 19.59 & 0.01 \\
\hline WD Teff & $\mathrm{K}$ & 13,247 & 200 \\
WD log $g$ & $\mathrm{dex}$ & 7.680 & 0.075 \\
WD cooling age & $\mathrm{Gyr}$ & 0.40 & 0.09 \\
WD mass & $\mathrm{M}_{\odot}$ & 0.4477 & 0.0052 \\
\hline $\mathrm{K}_{\text {sd }}$ & $\mathrm{km} \mathrm{s}^{-1}$ & 296.5 & 3.3 \\
$\gamma_{\text {sd }}$ & $\mathrm{kms}^{-1}$ & -117.7 & 2.7 \\
sd T eff & $\mathrm{K}$ & 3,650 & 50 \\
sd bolometric luminosity & $\mathrm{L}_{\odot}$ & $4.3 \times 10^{-3}$ & $2.4 \times 10^{-3}$ \\
sd spectral sub-type & & $\mathrm{usdK} 7$ & \pm 1 subclass \\
sd [Fe/H] abundance & $\mathrm{dex}$ & -1.55 & 0.25 \\
sd mass & $\mathrm{M}_{\odot}$ & 0.1501 & 0.0017 \\
sd radius (towards WD) & $\mathrm{R}_{\odot}$ & 0.1821 & 0.0007 \\
sd volume-averaged radius & $\mathrm{R}_{\odot}$ & 0.1669 & 0.0007 \\
\hline & & &
\end{tabular}

Table 1. Fitted and derived stellar and binary parameters for SDSS J2355+0448. sd and WD stand for cool subdwarf and white dwarf, respectively. We also include the right ascension and declination of the binary, the SDSS $g$-band magnitude, the Gaia parallax and the distance.

In most cases, the best-fitted template of the cool subdwarf was that of an sdK7 spectral type, although the sdM1 and sdM3 templates also provided good fits to some of the spectra. We emphasise that, at this stage, we are not aiming at determining the spectral type of the cool subdwarf, but only the stellar parameters of the white dwarf by subtracting the cool subdwarf flux contribution. The white dwarf effective temperatures obtained in this way from our X-Shooter optical individual spectra range from $12,882 \pm 29 \mathrm{~K}$ to $13,335 \pm 94 \mathrm{~K}$ and the surface gravities between $7.59 \pm 0.03$ and $7.76 \pm 0.03$ dex. Taking the average values we find $13,247 \pm 76 \mathrm{~K}$ and $7.68 \pm 0.03 \mathrm{dex}$, respectively. It has to be noted however that the uncertainties obtained are underestimated due to systematic errors in flux calibration, in the normalization process and/or in the model atmosphere grid used. Hence, we take more conservative uncertainties of $200 \mathrm{~K}$ and $0.075 \mathrm{dex}$ in our spectroscopic values of effective temperature and surface gravity, respectively. For completeness, we interpolated the averaged values of effective temperature and surface gravity in white dwarf cooling sequences for low metallicites ${ }^{18}$ to derive a (model dependent) spectroscopic white dwarf mass of $0.457 \pm 0.015 \mathrm{M}_{\odot}$ and a cooling age of $0.40 \pm 0.09$ Gyr. The spectroscopic white dwarf mass is in agreement with the mass obtained from our light-curve fitting method (see Table 1).

\section{The radial-velocity curve of the cool subdwarf}

We determined the radial velocities of the cool subdwarf by fitting a second-order polynomial plus a triple-Gaussian line profile to the Ca II absorption triplet at $\sim 8500 \AA$ sampled by our optical X-Shooter spectra (see the Methods section for a full description of the procedure). Periodograms calculated from the radial velocities to investigate the periodic nature of the velocity variations displayed a clear peak at $11.139 \mathrm{~d}^{-1}$ as well as a couple of weaker aliases due to the sampling pattern of 
the observations (Figure 2, top panel). We carried out sine-fits of the form

$$
V_{\mathrm{r}}=K_{\mathrm{sd}} \sin \left[\frac{2 \pi\left(t-T_{0}\right)}{P_{\mathrm{orb}}}\right]+\gamma
$$

to the radial-velocity data set, where $\gamma$ is the systemic velocity, $K_{\mathrm{sd}}$ is the radial velocity semi-amplitude of the cool subdwarf, $T_{0}$ is the time of inferior conjunction of the cool subdwarf, and $P_{\mathrm{orb}}$ is the orbital period. The sine-fit assumed the frequency corresponding to the strongest peak in the power spectrum as the orbital period of the binary, i.e. 2.154 hours, in agreement with the orbital period measured from the Catalina Sky Survey ${ }^{20}$ light curves. The radial-velocity curve folded over the orbital period is illustrated in the bottom panel of Figure 2.
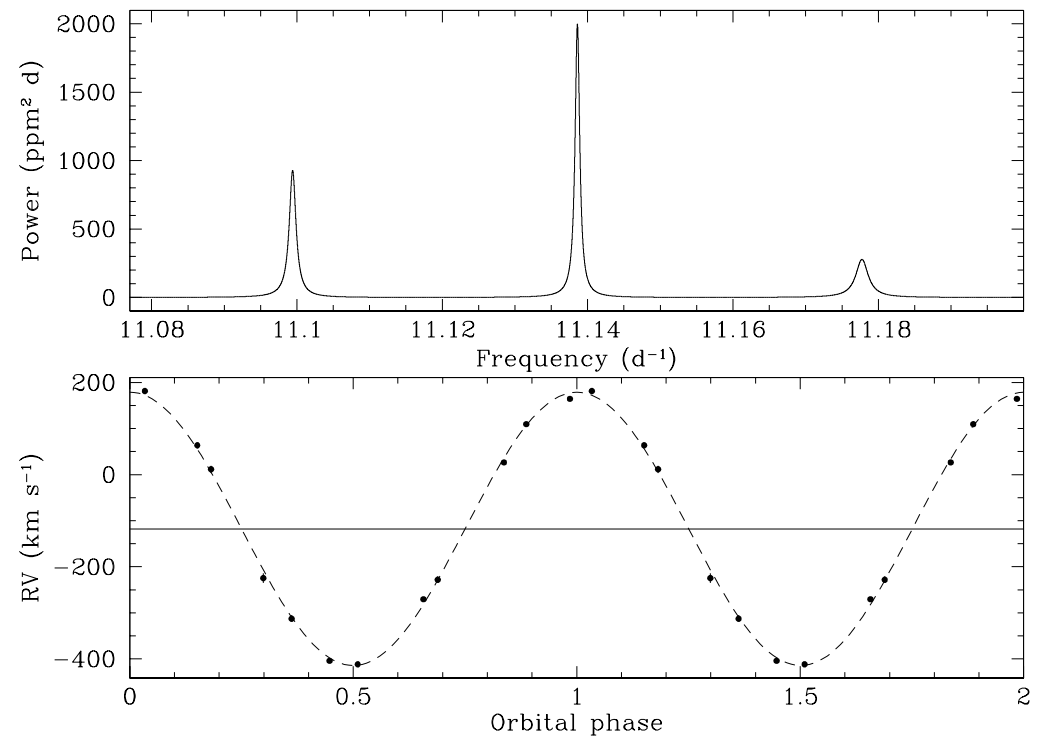

Figure 2. Periodogram obtained from the radial-velocity data of the cool subdwarf in the binary SDSS J2355+0448, derived from X-Shooter spectroscopy. A clear peak at $11.139 \mathrm{~d}^{-1}$ can be seen. Bottom panel: the radial-velocity curve folded over the period provided by the periodogram in the top panel. The radial-velocity $\pm 1 \sigma$ error bars are shown but are too small to see.

\section{The mass and radius of the stellar components}

The high-speed light curves of SDSS 2355+0448 (see Figure 3) revealed a sharp eclipse of the white dwarf, with an ingress/egress lasting 80 seconds and a total eclipse duration of 11 minutes. As was already apparent from the spectroscopy, the white dwarf dominates the overall flux in the $u_{s}$ and $g_{s}$ bands, resulting in a deep eclipse and little out-of-eclipse variation. At longer wavelengths the tidally distorted shape of the cool subdwarf results in a double-peaked ellipsoidal modulation signal away from the eclipse. In the $i_{s}$ band we detect the secondary eclipse (Figure 3). All of these features allowed us to constrain the stellar and binary parameters with minimal use of theoretical models.

We fitted the light curves using the LCURVE code $^{11}$, which is specifically designed for fitting the light curves of compact binary systems (see the Methods section for a detailed description of the light curve model and fitting procedure). Due to the lack of a direct measurement of the radial-velocity semi-amplitude of the white dwarf we were unable to determine the masses and radii of both stars completely independent of theoretical models. We therefore forced the radius of the white dwarf to follow a theoretical mass-radius relationship when fitting the light curve, hence the parameters of the cool subdwarf are only dependent upon the well tested white dwarf mass-radius relationship ${ }^{16}$.

\section{The effective temperature and the bolometric luminosity of the cool subdwarf star}

The Virtual Observatory SED Analyzer (VOSA) ${ }^{28}$ is a tool that compares observed photometry, gathered from a significant number of compliant VO catalogues, to different collections of theoretical model spectra for measuring the stellar parameters (effective temperature, surface gravity, metallicity, bolometric luminosity) of a given object/s. We used VOSA to derive the effective temperature and bolometric luminosity of the cool subdwarf in SDSS J2355+0448. A description of the methodology employed by VOSA is provided in the Methods section. 


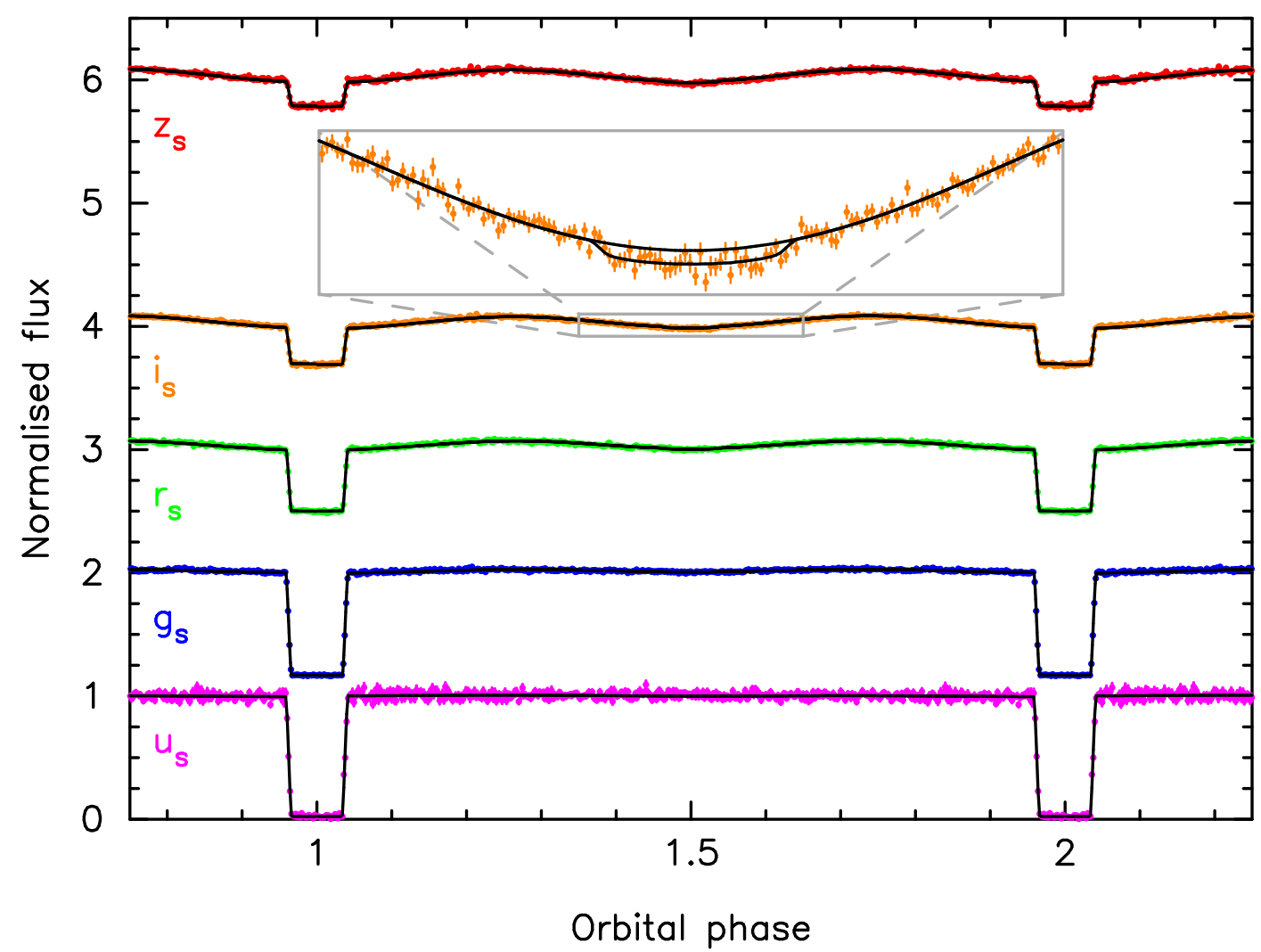

Figure 3. Phase-folded HiPERCAM light curves of SDSS J2355+0448 with model fits over-plotted (black lines). The $g_{s}, r_{s}$, $i_{s}$ and $z_{s}$ band data have been binned by a factor of three and offset vertically for clarity. We show a zoom-in to the $i_{s}$ band light-curve, which displays the secondary eclipse (i.e. the transit of the white dwarf in front of the cool subdwarf). Over-plotted is our best fit model with the secondary eclipse turned on and off to demonstrate its depth. The error bars represent $\pm 1 \sigma$ uncertainties.

Given that the white dwarf and the cool subdwarf are spatially unresolved, we did not make use of VOSA to gather available optical photometry, since those values correspond to the magnitudes of the binary system and not to the cool subdwarf. Hence, we derived the optical magnitudes of the cool subdwarf from our HiPERCAM light curves during eclipse, when the flux contribution from the white dwarf is zero. The HiPERCAM magnitudes were converted into SDSS ugriz photometry. Colour terms between the HiPERCAM and the SDSS photometric systems were determined by folding main-sequence template spectra ${ }^{29}$ through theoretical band-passes for HiPERCAM. We also considered the publicly available near-infrared $h k$ UKIDSS $^{30}$ magnitudes of SDSS J2355+0448, where the white dwarf flux contribution is negligible (the $y j$ magnitudes do show signs of contamination from the flux of the white dwarf, as revealed from the X-Shooter residual cool subdwarf spectrum that results from subtracting the best-fit white dwarf model spectrum; see Fig 4) and the far-infrared WISE ${ }^{31} w_{1} w_{2}$ magnitudes, where the white dwarf flux contribution is also negligible. For each catalogue we adopted a search radius of 3". No $2 \mathrm{MASS}^{32}$, VISTA ${ }^{33}$ or extra infra-red magnitudes from other surveys were found for our target. In this way we built the observational SED of the cool subdwarf from the optical to the far-infrared wavelength range.

Two physical parameters were obtained from the VOSA SED fits, the effective temperature and the bolometric luminosity. The effective temperature was constrained to be $3600-3700 \mathrm{~K}$, depending on the adopted model. Considering that the intrinsic error provided by VOSA is $50 \mathrm{~K}$, we assumed a value of $3650 \pm 50 \mathrm{~K}$ for the cool subdwarf. The luminosities varied from $(4.2 \pm 2.4) \times 10^{-3} \mathrm{~L}_{\odot}$ to $(4.4 \pm 2.4) \times 10^{-3} \mathrm{~L}_{\odot}$ and we thus adopted a value of $(4.3 \pm 2.4) \times 10^{-3} \mathrm{~L} \odot$. The large luminosity uncertainty is directly related to the distance error. These calculated values of effective temperature and bolometric luminosity translate into a radius of $0.164 \pm 0.050 \mathrm{R}_{\odot}$ from the Stefan-Boltzmann equation, in good agreement with the radius derived from the light-curve fitting $\left(0.1669 \pm 0.0007 \mathrm{R}_{\odot}\right)$.

For illustrative purposes, in Figure 4 we show the observational SED built together with the synthetic spectrum that best fits the photometric data. The synthetic spectrum is an excellent match to the observed SED. 


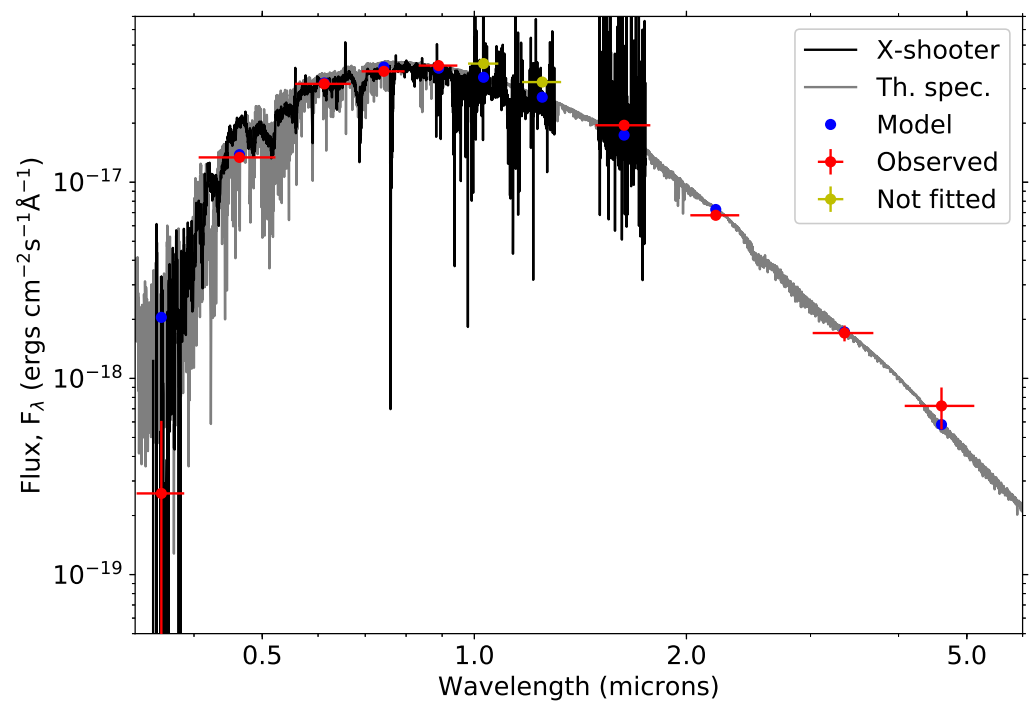

Figure 4. The observational SED of the cool subdwarf in SDSS J2355+0448 (black line, note the white dwarf contribution has been subtracted) and its best-fit model performed by VOSA (gray line). The observational photometric points are shown in red (optical points are obtained by us directly from the light curve during white dwarf eclipse) and the blue dots are the synthetic photometry. The yellow dots are UKIDSS $y j$ photometry that have not been used in the fit due to white dwarf flux contamination. The error bars represent $\pm 1 \sigma$ uncertainties.

\section{The metallicity class and the $[\mathrm{Fe} / \mathrm{H}]$ abundance of the cool subdwarf star}

The parameter $\tau_{\mathrm{TiO} / \mathrm{CaH}}{ }^{6,34}$ is a good indicator of the metallicity class of a dwarf star. It is based on the flux ratio at specific wavelength ranges and quantifies the weakening of the TiO band strength due to the effect of metallicity. In order to calculate a reliable value of this ratio for the cool subdwarf, we subtracted the white dwarf contribution from the X-Shooter spectrum of SDSS J2355+0448. In this case, since we have measured the radial velocities of the cool subdwarf, we were able to correct the orbital motion of the star and hence combine all spectra to one single (averaged) spectrum. To subtract the white dwarf's flux contribution we considered a white dwarf model atmosphere spectrum of the same effective temperature and surface gravity values as derived for the white dwarf in this system (see Table 1), obtained by interpolating these parameters in our full white dwarf model atmosphere grid ${ }^{7}$ and scaled in flux. We thus calculated a value of $\tau_{\mathrm{TiO} / \mathrm{CaH}}=0.095$ from the residual cool subdwarf spectrum, i.e. an ultra-low metallicity usd spectral class ${ }^{6,34}$. Using three different calibrators, this translates into an iron abundance of $[\mathrm{Fe} / \mathrm{H}]=-1.6 \pm 0.2 \mathrm{dex}^{5},-1.1 \pm 0.2 \mathrm{dex}^{35}$ and $-1.5 \pm 0.3 \mathrm{dex}^{7}$. The possible value for the $[\mathrm{Fe} / \mathrm{H}]$ abundance of the cool subdwarf ranges then from -0.9 to -1.8 dex. However, a usd metallicity class implies $[\mathrm{Fe} / \mathrm{H}]<-1.35$ $\operatorname{dex}^{7}$ and we hence assume the $[\mathrm{Fe} / \mathrm{H}]$ abundance should be within -1.35 and -1.8 dex, i.e. $[\mathrm{Fe} / \mathrm{H}]=-1.55 \pm 0.25$ dex. We also made use of iSpec ${ }^{25}$, an open source framework for spectral analysis, to attempt to determine the $[\mathrm{Fe} / \mathrm{H}]$ abundance of the cool subdwarf star. However, the value obtained is subject to large uncertainties $([\mathrm{Fe} / \mathrm{H}]=-2.1 \pm 2$ dex $)$ due to the relatively low signal to noise ratio as well as the intermediate resolution of our spectra (see the Methods section for details).

To determine the spectral sub-type of the usd star we re-applied our decomposition/fitting routine to the averaged XShooter optical spectrum, using a usd spectral library of 27 templates $^{6}$ instead. The spectral sub-type obtained in this way for the low-metallicity star is usdK7.

\section{The past and future evolution of SDSS J2355+0448}

Given its short orbital period, the binary star SDSS J2355+0448 has very likely evolved in the past through a common envelope phase $^{37}$. The young cooling age of the white dwarf implies such an event took place very recently and that for most of its life the binary star was very likely composed of two low-mass low-metallicity main-sequence stars. The low mass measured for the white dwarf implies the common envelope phase truncated the evolution of the white dwarf precursor when it was ascending the giant branch. Thus, the white dwarf core is expected to be composed mainly of helium. It has to be noted however that the common envelope phase is not expected to have modified the parameters of the cool subdwarf. This is because secondary stars are not envisaged to be able to accrete the overflowing material during a common envelope episode due to their long thermal time-scales ${ }^{29}$, a hypothesis that seems to be observationally confirmed ${ }^{20}$. Because the common envelope truncated the evolution of the white dwarf progenitor, we are not able to derive the white dwarf's progenitor mass using an initial-to-final mass relation for single stellar evolution ${ }^{40,41}$ nor derive a total age for the system. It is important to emphasise however that 
the current age of SDSS J2355+0448 is likely to be very old ( $210 \mathrm{Gyr}$ ) given the low metallicity of the usd star. Indeed, the space velocity with respect to the local standard of rest $(\mathrm{U}, \mathrm{V}, \mathrm{W})=(-36.5 \pm 29.3,-208.3 \pm 24.8,3.7 \pm 17.3) \mathrm{km} / \mathrm{s}$ derived from Gaia data adopting the systemic velocity we measured for the binary is consistent with halo membership for this object, although we cannot rule out a thick disk origin ${ }^{11}$. Due to the short orbital period, the cool subdwarf in SDSS J2355+0448 is tidally locked to the white dwarf (the ellipsoidal modulation can clearly be seen in Figure 3), therefore its rotational period is commensurate with the orbital period. This fast rotation induces chromospheric activity on the cool subdwarf (see a light-curve displaying a flare in Supplementary Figure 2).

We used the binary stellar evolution code binary_c $c^{27,28}$ (see a full description in the Methods section), based on the BSE $\operatorname{code}^{29}$, to predict the future evolution of SDSS J2355+0448. The results obtained indicate that a phase of stable mass transfer will ensue in $\simeq 800 \mathrm{Myr}$. The system will then become a semi-detached cataclysmic variable. We note however that the evolutionary timescale prior to the onset of mass transfer should be taken with caution since the angular momentum loss mechanism/s that bring the two stars closer are not yet fully understood.

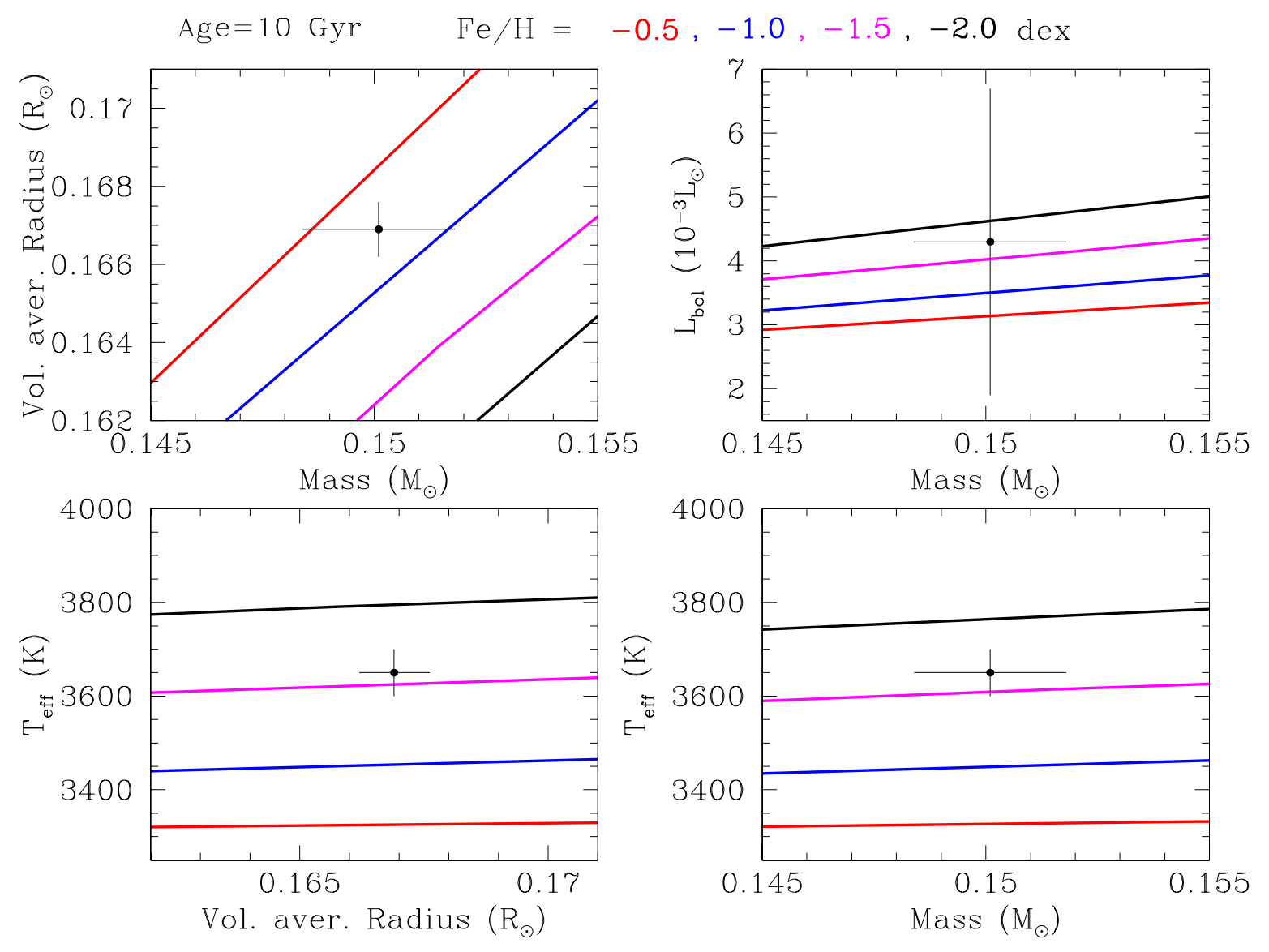

Figure 5. The observed stellar parameter relations of the cool subdwarf in SDSS J2355+0448 (black solid dots; the error bars represent $\pm 1 \sigma$ uncertainties) as compared to the theoretical relations ${ }^{44,45}$ for an age of $10 \mathrm{Gyr}$ and $[\mathrm{Fe} / \mathrm{H}]$ values from -0.5 to -2.0 dex (solid lines).

\section{Discussion}

We show a comparison between the observed parameter relations for the cool subdwarf in SDSS J2355+0448 and those expected from the theoretical Dartmouth isochrones ${ }^{44,45}$ in Figure 5. Inspection of the Figure reveals that the theoretical relations agree well with our measured values when assuming an $[\mathrm{Fe} / \mathrm{H}]$ abundance of $-1.5 \mathrm{dex}$ (a value which is in excellent agreement with our estimate of $-1.55 \pm 0.25 \mathrm{dex}$ ), except for the theoretical mass-radius relation, which yields a slightly overestimated radius for the measured mass. This could be due to uncertainties in the adopted limb-darkening coefficients for the cool subdwarf when performing our light-curve fit (see the Methods section for further details). Alternatively, an over-sized radius could be a consequence of the cool subdwarf star being magnetically active (see Supplementary Figure 2). 
Over-inflated radii are often measured for magnetically active low-mass main-sequence stars of solar metallicity ${ }^{20}$. It is also important to note that the measured radius of the cool subdwarf is undersized compared to solar metallicity models ${ }^{45,46}$, which is consistent with the theory that cool subdwarfs should have smaller radii for a given effective temperature (see Supplementary Figure 3).

In this paper we have studied in detail the cool subdwarf in the eclipsing binary SDSS J2355+0448. The precise mass and radius we have determined for this star together with the effective temperature and luminosity we have calculated have allowed us to validate the theoretical models of low-mass low-metallicity stars.

\section{References}

1. Burrows, A., Hubbard, W. B., Saumon, D. \& Lunine, J. I. An expanded set of brown dwarf and very low mass star models. Astrophys. J. 406, 158-171, DOI: 10.1086/172427 (1993).

2. Morgan, W. W., Keenan, P. C. \& Kellman, E. An atlas of stellar spectra, with an outline of spectral classification (The University of Chicago press, 1943).

3. Burgasser, A. J. et al. The First Substellar Subdwarf? Discovery of a Metal-poor L Dwarf with Halo Kinematics. Astrophys. J. 592, 1186-1192, DOI: 10.1086/375813 (2003). astro-ph/0304174.

4. Digby, A. P., Hambly, N. C., Cooke, J. A., Reid, I. N. \& Cannon, R. D. The subdwarf luminosity function. Mon. Not. R. Astron. Soc. 344, 583-601, DOI: 10.1046/j.1365-8711.2003.06842.x (2003). astro-ph/0304056.

5. Rajpurohit, A. S. et al. High-resolution spectroscopic atlas of M subdwarfs. Effective temperature and metallicity. Astron. Astrophys. 564, A90, DOI: 10.1051/0004-6361/201322881 (2014). 1401.2901.

6. Lépine, S., Rich, R. M. \& Shara, M. M. Revised Metallicity Classes for Low-Mass Stars: Dwarfs (dM), Subdwarfs (sdM), Extreme Subdwarfs (esdM), and Ultrasubdwarfs (usdM). Astrophys. J. 669, 1235-1247, DOI: 10.1086/521614 (2007). 0707.2993.

7. Woolf, V. M., Lépine, S. \& Wallerstein, G. Calibrating M-Dwarf Metallicities Using Molecular Indices: Extension to Low-metallicity Stars. Publ. Astron. Soc. Pac. 121, 117, DOI: 10.1086/597433 (2009).

8. Gizis, J. E. M-Subdwarfs: Spectroscopic Classification and the Metallicity Scale. Astron. J. 113, 806-822, DOI: 10.1086/118302 (1997). astro-ph/9611222.

9. Stoughton, C. et al. Sloan Digital Sky Survey: Early Data Release. Astron. J. 123, 485-548, DOI: 10.1086/324741 (2002).

10. Cui, X.-Q. et al. The Large Sky Area Multi-Object Fiber Spectroscopic Telescope (LAMOST). Res. Astron. Astrophys. 12, 1197-1242, DOI: 10.1088/1674-4527/12/9/003 (2012).

11. Savcheva, A. S., West, A. A. \& Bochanski, J. J. A New Sample of Cool Subdwarfs from SDSS: Properties and Kinematics. Astrophys. J. 794, 145, DOI: 10.1088/0004-637X/794/2/145 (2014). 1409.1229.

12. Bai, Y. et al. Nearby M subdwarfs from LAMOST data release 2. Res. Astron. Astrophys. 16, 107, DOI: 10.1088/1674-4527/16/7/107 (2016).

13. Kesseli, A. Y. et al. Radii of 88 M Subdwarfs and Updated Radius Relations for Low-Metallicity M Dwarf Stars. ArXiv e-prints (2018). 1810.07702.

14. Jao, W.-C., Nelan, E. P., Henry, T. J., Franz, O. G. \& Wasserman, L. H. Cool Subdwarf Investigations. III. Dynamical Masses of Low-metallicity Subdwarfs. Astron. J. 152, 153, DOI: 10.3847/0004-6256/152/6/153 (2016). 1607.01304.

15. Kepler, S. O. et al. New white dwarf stars in the Sloan Digital Sky Survey Data Release 10. Mon. Not. R. Astron. Soc. 446, 4078-4087, DOI: 10.1093/mnras/stu2388 (2015). 1411.4149.

16. Rebassa-Mansergas, A. et al. The SDSS spectroscopic catalogue of white dwarf-main-sequence binaries: new identifications from DR 9-12. Mon. Not. R. Astron. Soc. 458, 3808-3819, DOI: 10.1093/mnras/stw554 (2016). 1603.01017.

17. Ren, J.-J. et al. White dwarf-main sequence binaries from LAMOST: the DR5 catalogue. Mon. Not. R. Astron. Soc. 477, 4641-4654, DOI: 10.1093/mnras/sty805 (2018). 1803.09523.

18. Parsons, S. G. et al. Testing the white dwarf mass-radius relationship with eclipsing binaries. Mon. Not. R. Astron. Soc. 470, 4473-4492, DOI: 10.1093/mnras/stx1522 (2017). 1706.05016.

19. Parsons, S. G. et al. The scatter of the M dwarf mass-radius relationship. Mon. Not. R. Astron. Soc. 481, 1083-1096, DOI: 10.1093/mnras/sty2345 (2018). 1808.07780. 
20. Drake, A. J. et al. First Results from the Catalina Real-Time Transient Survey. Astrophys. J. 696, 870-884, DOI: 10.1088/0004-637X/696/1/870 (2009). 0809.1394.

21. Dhillon, V. et al. First light with HiPERCAM on the GTC. In Society of Photo-Optical Instrumentation Engineers (SPIE) Conference Series, vol. 10702 of Society of Photo-Optical Instrumentation Engineers (SPIE) Conference Series, 107020L, DOI: 10.1117/12.2312041 (2018). 1807.00557.

22. Rebassa-Mansergas, A., Gänsicke, B. T., Rodríguez-Gil, P., Schreiber, M. R. \& Koester, D. Post-common-envelope binaries from SDSS - I. 101 white dwarf main-sequence binaries with multiple Sloan Digital Sky Survey spectroscopy. Mon. Not. R. Astron. Soc. 382, 1377-1393, DOI: 10.1111/j.1365-2966.2007.12288.x (2007). 0707.4107.

23. Rajpurohit, A. S. et al. Spectral energy distribution of M-subdwarfs: A study of their atmospheric properties. Astron. Astrophys. 596, A33, DOI: 10.1051/0004-6361/201526776 (2016). 1609.07062.

24. Koester, D. White dwarf spectra and atmosphere models. Memorie della Soc. Astron. Italiana 81, 921-931 (2010).

25. Bailer-Jones, C. A. L., Rybizki, J., Fouesneau, M., Mantelet, G. \& Andrae, R. Estimating Distance from Parallaxes. IV. Distances to 1.33 Billion Stars in Gaia Data Release 2. Astron. J. 156, 58, DOI: 10.3847/1538-3881/aacb21 (2018). 1804.10121.

26. Althaus, L. G., De Gerónimo, F., Córsico, A., Torres, S. \& García-Berro, E. The evolution of white dwarfs resulting from helium-enhanced, low-metallicity progenitor stars. Astron. Astrophys. 597, A67, DOI: 10.1051/0004-6361/201629909 (2017). 1611.06191.

27. Copperwheat, C. M. et al. Physical properties of IP Pegasi: an eclipsing dwarf nova with an unusually cool white dwarf. Mon. Not. R. Astron. Soc. 402, 1824-1840, DOI: 10.1111/j.1365-2966.2009.16010.x (2010). 0911.1637.

28. Bayo, A. et al. VOSA: virtual observatory SED analyzer. An application to the Collinder 69 open cluster. Astron. Astrophys. 492, 277-287, DOI: 10.1051/0004-6361:200810395 (2008). 0808.0270.

29. Pickles, A. J. A Stellar Spectral Flux Library: 1150-25000 Å. Publ. Astron. Soc. Pac. 110, 863-878, DOI: 10.1086/316197 (1998).

30. Hewett, P. C., Warren, S. J., Leggett, S. K. \& Hodgkin, S. T. The UKIRT Infrared Deep Sky Survey ZY JHK photometric system: passbands and synthetic colours. Mon. Not. R. Astron. Soc. 367, 454-468, DOI: 10.1111/j.1365-2966.2005.09969.x (2006). astro-ph/0601592.

31. Wright, E. L. et al. The Wide-field Infrared Survey Explorer (WISE): Mission Description and Initial On-orbit Performance. Astron. J. 140, 1868-1881, DOI: 10.1088/0004-6256/140/6/1868 (2010). 1008.0031.

32. Skrutskie, M. F. et al. The Two Micron All Sky Survey (2MASS). Astron. J. 131, 1163-1183, DOI: 10.1086/498708 (2006).

33. Cross, N. J. G. et al. The VISTA Science Archive. Astron. Astrophys. 548, A119, DOI: 10.1051/0004-6361/201219505 (2012). 1210.2980.

34. Lépine, S. et al. A Spectroscopic Catalog of the Brightest $(\mathrm{J}<9)$ M Dwarfs in the Northern Sky. Astron. J. 145, 102, DOI: 10.1088/0004-6256/145/4/102 (2013). 1206.5991.

35. Mann, A. W., Brewer, J. M., Gaidos, E., Lépine, S. \& Hilton, E. J. Prospecting in Late-type Dwarfs: A Calibration of Infrared and Visible Spectroscopic Metallicities of Late K and M Dwarfs Spanning 1.5 dex. Astron. J. 145, 52, DOI: 10.1088/0004-6256/145/2/52 (2013). 1211.4630.

36. Blanco-Cuaresma, S., Soubiran, C., Heiter, U. \& Jofré, P. Determining stellar atmospheric parameters and chemical abundances of FGK stars with iSpec. Astron. Astrophys. 569, A111, DOI: 10.1051/0004-6361/201423945 (2014). 1407.2608.

37. Iben, I., Jr. \& Livio, M. Common envelopes in binary star evolution. Publ. Astron. Soc. Pac. 105, 1373-1406, DOI: 10.1086/133321 (1993).

38. Hurley, J. R., Tout, C. A. \& Pols, O. R. Evolution of binary stars and the effect of tides on binary populations. Mon. Not. R. Astron. Soc. 329, 897-928, DOI: 10.1046/j.1365-8711.2002.05038.x (2002). astro-ph/0201220.

39. Parsons, S. G. et al. The scatter of the M dwarf mass-radius relationship. Mon. Not. R. Astron. Soc. 481, 1083-1096, DOI: 10.1093/mnras/sty2345 (2018). 1808.07780.

40. Catalán, S., Isern, J., García-Berro, E. \& Ribas, I. The initial-final mass relationship of white dwarfs revisited: effect on the luminosity function and mass distribution. Mon. Not. R. Astron. Soc. 387, 1693-1706, DOI: 10.1111/j.1365-2966.2008.13356.x (2008). 0804.3034. 
41. Cummings, J. D., Kalirai, J. S., Tremblay, P.-E., Ramirez-Ruiz, E. \& Choi, J. The White Dwarf Initial-Final Mass Relation for Progenitor Stars from 0.85 to 7.5 M $\odot$. Astrophys. J. 866, 21, DOI: 10.3847/1538-4357/aadfd6 (2018). 1809.01673.

42. Izzard, R. G., Tout, C. A., Karakas, A. I. \& Pols, O. R. A new synthetic model for asymptotic giant branch stars. Mon. Not. R. Astron. Soc. 350, 407-426, DOI: 10.1111/j.1365-2966.2004.07446.x (2004). astro-ph/0402403.

43. Izzard, R. G. et al. Binary stars in the Galactic thick disc. Mon. Not. R. Astron. Soc. 473, 2984-2999, DOI: 10.1093/mnras/stx2355 (2018). 1709.05237.

44. Dotter, A. et al. The Dartmouth Stellar Evolution Database. Astrophys. J. 178, 89-101, DOI: 10.1086/589654 (2008). 0804.4473.

45. Feiden, G. A., Chaboyer, B. \& Dotter, A. Accurate Low-mass Stellar Models of KOI-126. Astrophys. J. 740, L25, DOI: 10.1088/2041-8205/740/1/L25 (2011). 1109.2063.

46. Baraffe, I., Chabrier, G., Allard, F. \& Hauschildt, P. H. Evolutionary models for metal-poor low-mass stars. Lower main sequence of globular clusters and halo field stars. Astron. Astrophys. 327, 1054-1069 (1997). astro-ph/9704144.

\section{Methods}

\section{Observations and their reduction}

SDSS J2355+0448 was observed with the medium resolution echelle spectrograph X-Shooter ${ }^{1}$ on the $8.2 \mathrm{~m}$ Very Large Telescope (VLT) in Chile on the nights of the 12th of June 2018 and the 8th of July 2018. X-Shooter consists of three detectors (UVB, VIS and NIR) which obtain simultaneous spectra from the ultraviolet atmospheric cutoff ( $\sim 3000 \AA$ ) to the K band ( 2.5 microns). SDSS J2355+0448 was observed for a full binary orbit on both nights, recording a total of 12 UVB and VIS spectra and 28 NIR spectra. Exposure times were kept short (1073s, 1088s, 480s in the UVB, VIS and NIR arms respectively) to minimise the amount of orbital smearing of the lines. Due to the faintness of the binary in the near-infrared $(J=17.5)$ the NIR arm spectra have very low signal-to-noise and were not used in any subsequent analysis. The data were reduced using the latest release of the X-Shooter reduction pipeline (version 2.9.3). Standard reduction steps were used to debias, flat-field, extract and wavelength calibrate the data. The spectra were flux calibrated using spectra of the spectrophotometric standard star Feige 110 obtained in twilight at the end of both nights. Finally, all spectra were placed on a heliocentric wavelength scale.

High-speed multi-band photometry of SDSS J2355+0448 was obtained using HiPERCAM ${ }^{2}$ mounted on the $10.4 \mathrm{~m}$ Gran Telescopio Canarias (GTC). HiPERCAM is a quintuple-beam imager equipped with frame-transfer CCDs allowing simultaneous $u, g, r, i$ and $z$ band imaging at frames rates of up to 1000 frames per second. Note that HiPERCAM uses modified versions of the SDSS filters with much higher throughput, known as Super-SDSS filters. These are denoted as $u_{s} g_{s} r_{s} i_{s} z_{s}$ to distinguish them from the standard SDSS filters (ugriz). Exposure times of $5 \mathrm{~s}$ were used in the $g_{s}, r_{s}, i_{s}$ and $z_{s}$ bands and $15 \mathrm{~s}$ in the $u_{s}$ band. SDSS J2355+0448 was observed for a full orbit on the night of the 5th of October 2018 , and 50 minutes of data were obtained around the secondary eclipse on the nights of the 31st of October 2018 and 1st of November 2018 . The data were reduced using the dedicated HiPERCAM pipeline, including debiasing, flat-fielding and fringe correction in the $z_{s}$ band. Differential photometry was performed using the nearby star SDSS J235517.21+045057.1 as a reference source. All times were converted to the barycentric dynamical timescale, corrected to the solar system barycentre, MJD(BTDB).

\section{The spectral decomposition/fitting technique}

The decomposition/fitting routine used in this work ${ }^{3}$ was developed to derive spectroscopic stellar parameters of white dwarf plus $\mathrm{M}$ dwarf binaries, namely the spectral sub-type of the $\mathrm{M}$ dwarf and its radius, and the effective temperature ( $\mathrm{T}_{\mathrm{eff}}$ ), surface gravity $(\log g)$, mass and radius of the white dwarf. It uses an evolution strategy ${ }^{4}$ to decompose the observed spectrum into the two stellar components. To that end it optimises a fitness function (i.e. a weighted $\chi^{2}$ ). The only difference between the original routine and the one employed in this work is adopting a series of cool subdwarf template spectra ${ }^{5,6}$ instead of the original $10 \mathrm{M}$ dwarf templates.

The routine fits first the entire spectrum and selects the best-fit cool subdwarf template, which is scaled in flux and subtracted. Consequently, the residual white dwarf spectrum is fitted with a grid of hydrogen-rich white dwarf model atmosphere spectra $^{7}$. The grid includes 1,339 spectra covering the 6,000-100,000 K $\mathrm{T}_{\text {eff }}$ and the $6.5-9.5$ dex $\log g$ values. The fit is performed to the normalised $\mathrm{H} \beta$ to $\mathrm{H} 10$ Balmer lines (the $\mathrm{H} \alpha$ line is excluded in this process since it covers a wavelength range of largest residual contamination from the cool subdwarf). A bi-cubic spline interpolation to the $\chi^{2}$ values on the $\mathrm{T}_{\mathrm{eff}}-\log g$ grid is used to derive the best-fit $\mathrm{T}_{\text {eff }}$ and $\log g$. The errors ( $1 \sigma$ uncertainties) are calculated projecting the $\Delta \chi^{2}=1$ contour around the $\chi^{2}$ of the best fit into the $\mathrm{T}_{\text {eff }}$ and $\log g$ axes. This results in a range of parameters which are averaged into symmetric error bars (see the top panels of Supplementary Figure 1). 
Since the equivalent widths of the Balmer lines of white dwarfs reach a maximum for effective temperatures near $13000 \mathrm{~K}$, the Balmer line profile fits provide two sets of $\mathrm{T}_{\text {eff }}$ and $\log g$ values, which are generally known as the "hot" and "cold" solutions. In other words, similar fits can be achieved on both temperature sides at which the maximum equivalent width takes place. To break this degeneracy the routine makes use of the model spectra grid for fitting the entire spectrum over the wavelength range $3850-7150 \AA$ (see the bottom panel of Supplementary Figure 1). The red part of the spectrum is not considered by the fit due to possible distortions in the residual white dwarf spectrum arising from subtraction of the cool subdwarf. The $\mathrm{T}_{\text {eff }}$ and $\log g$ values derived from the fit to the whole spectrum are used to discard one of the "hot"/"cold" solutions. In the case of SDSS J2355+0448, the best-fit $\mathrm{T}_{\text {eff }}$ and $\log g$ from the whole spectrum agrees with the one obtained by the "hot" solution (see the top-right panel of Supplementary Figure 1).

\section{The radial-velocity fitting method and the periodogram}

We fitted the $\mathrm{Ca}_{\text {II }}$ absorption triplet at $\sim 8500 \AA$ sampled by the X-Shooter VIS arm spectra with a triple-Gaussian absorption profile of fixed separation (see an example in Supplementary Figure 4). To that end we fixed the width of each Gaussian, however we set the three amplitudes and the radial velocity of the cool subdwarf star as free parameters. This fitting method has been shown to provide both accurate and precise $\mathrm{M}$ dwarf radial velocities in binary systems with white dwarf companions such as the one studied in this work ${ }^{8}$.

We run a Lomb-Scargle periodogram ${ }^{9}$ to the radial velocities of the cool subdwarf in SDSS J2355+0448, which unfortunately contained several aliases as a consequence of the sampling pattern of the observations. We thus tried the ORT/TSA ${ }^{10}$ command in MIDAS, which provided a periodogram with a clear pick at $11.139 \mathrm{~d}^{-1}$. The ORT/TSA routine uses a grid of trial periods to fold and phase-bin the data and subsequently fits the folded radial velocity curve with a series of Fourier terms.

\section{The light-curve fitting method}

We fitted the HiPERCAM light curves of SDSS J2355+0448 (see Figure 3) using a code written for binaries containing white dwarfs ${ }^{11,12}$. Each stellar component in the binary is subdivided into small elements by the program. This is performed by following a geometry that is fixed by the radius of the considered star as measured along the direction of centres towards the other star. The code takes into account limb- and gravity-darkening, gravitational microlensing, Roche geometry distortion, irradiation effects and eclipses. It uses a non-linear four-parameter model to implement limb-darkening ${ }^{13}$, of the form

$$
\frac{I(\mu)}{I(1)}=1-\sum_{k=1}^{4} a_{k}\left(1-\mu^{\frac{k}{2}}\right)
$$

being $\mu=\cos \phi$ (where $\phi$ is the angle between the emergent flux and the line of sight), and $I(1)$ the monochromatic specific intensity at the centre of the stellar disk. Supplementary Table 1 lists the adopted limb-darkening coefficients for the white dwarf ${ }^{14}$ and cool subdwarf ${ }^{15}$.

The model requires a set of additional parameters to be defined, namely the binary inclination $i$, the mass ratio $q=$ $M_{\mathrm{sd}} / M_{\mathrm{WD}}$, the orbital period $P_{\mathrm{orb}}$, the sum of the unprojected stellar orbital speeds $V_{s}=\left(K_{\mathrm{WD}}+K_{\mathrm{sd}}\right) / \sin i$, the mid-time when the white dwarf eclipse occurs $T_{0}$, the radii of the stars scaled by the orbital separation $R_{\mathrm{WD}} / a$ and $R_{\mathrm{sd}} / a$, and the temperatures of the stars $\mathrm{T}_{\text {eff,WD }}$ and $\mathrm{T}_{\text {eff,sd }}$ (is has to emphasised that temperatures are flux scaling parameters, therefore correspond only approximately to the real temperatures). All these parameters were considered as free when fitting the HiPERCAM light curves, the only exceptions were the white dwarf temperature, which was fixed at 13,250 K (Table 1) and the orbital period, which was fixed to the value found from the Catalina Sky Survey results. We phase-folded all of our HiPERCAM observations in order to combine data taken on different nights.

The information provided by the profile of the white dwarf eclipse is not sufficient to determine the radii of both stars as well as the orbital inclination. As a consequence, the fitting program requires additional information ${ }^{16}$ to break the degeneracy between the scaled radii and the inclination. This can be provided by the distorted shape of the cool subdwarf owing to its proximity to the white dwarf, which results in a double-peaked feature in the light-curve known as ellipsoidal modulation. Unfortunately, the fits to this double-peaked modulation highly depend on the adopted limb-darkening coefficients for the cool subdwarf as well as the presence of any star-spots. This is a particularly large problem in the case of the cool subdwarf star in SDSS J2355+0448 because of the uncertainty in its metallicity. Adopting the limb darkening parameters for a $[\mathrm{Fe} / \mathrm{H}]=-1$ dex or $[\mathrm{Fe} / \mathrm{H}]=-3$ dex star changes the resulting mass and radius values by 10 and 15 per cent from the $[\mathrm{Fe} / \mathrm{H}]=-2 \mathrm{dex}$ parameters (note that only these three $[\mathrm{Fe} / \mathrm{H}]$ abundance values are available for acquiring the limb-darkening coefficients ${ }^{15}$ ), meaning that fits relying on the amplitude of the ellipsoidal modulation are quite uncertain.

We managed to break the degeneracy between the scaled radii and the inclination by measuring the depth of the secondary eclipse (see Figure 3), an approach that allowed us to measure precise parameters for the stars. It has to be stressed that following this procedure considerably alleviates the dependence of the measured parameters with the adopted limb-darkening 
coefficients for the cool subdwarf star, i.e. the resulting masses and radii change by only 1.5 and 2 per cent in this case depending on the adopted value of $[\mathrm{Fe} / \mathrm{H}]$, comparable to our measured uncertainties. However, the lack of a direct determination of the radial velocity semi-amplitude of the white dwarf $K_{\mathrm{WD}}$ implied that we were not able to measure the masses and radii of the white dwarf and the cool subdwarf independently of any theoretical model. Fortunately, a recent observational study shows that the white dwarf measured radii in eclipsing binaries are in excellent agreement with theoretical expectations ${ }^{16}$. Therefore, we adopted the white dwarf's radius to follow the theoretical mass-radius relationship for a $13,250 \mathrm{~K}$ helium-core white dwarf with a thick surface hydrogen envelope ${ }^{17}$ when fitting the HiPERCAM light curves. We allowed some departure from the relation to account for a $500 \mathrm{~K}$ uncertainty in the white dwarf temperature. Note that the radii of very low metallicity $([\mathrm{Fe} / \mathrm{H}]<-3$ dex $)$ white dwarfs are 1-2 per cent larger than solar metallicity white dwarfs ${ }^{18}$, which is comparable to the departure allowed to account for the temperature uncertainty.

For determining the distributions of our model parameters we employed the Markov Chain Monte Carlo (MCMC) method ${ }^{19}$. We based the likelihood of accepting a model on a combination of the $\chi^{2}$ of the light-curve fit to the data and an additional prior probability whereby we ensure that $k_{\mathrm{sd}}$ is consistent with the spectroscopic result and that the white dwarf's radius is consistent with the mass-radius relationship outlined above. For each band, the approximate best parameters and covariances were determined using an initial MCMC chain. These approximate best parameters were then considered as the starting values for longer chains which were employed to derive the final model values and their uncertainties. We simultaneously run four chains in order to ensure that they converged on the same values, with the initial approximated best parameters slightly perturbed. From each chain, the 50,000 first points were classified as a "burn-in" phase and were removed for the subsequent analysis. Each chain had a total length of 250,000 points.

The full light curves and model fits are shown in Figure 3 . The parameters that result from the $u_{s}$ and $g_{s}$ band fits have associated large uncertainties since these bands are dominated by the white dwarf' flux and therefore contain relatively little information regarding the inclination. The secondary eclipse is only detected in the $i_{s}$ band and therefore the tightest constraints come from these data.

It is important to emphasise the light-curve fitting code provides both the tidally-distorted radius of the cool subdwarf (towards the white dwarf) as well as its volume-averaged radius (Table 1). We adopted the latter in our analysis, which is expected to make the effects of tidal distortion negligible when comparing the observed to the theoretical relations for this $\operatorname{star}^{20}$.

\section{The VOSA spectral energy distribution fitting routine}

Different statistical tests may be employed by VOSA for determining which model fits best the observational data. In our case, we used the $\chi^{2}$ statistical test between the model spectra and the observed photometry to derive the effective temperature and luminosity of the cool subdwarf. Good fits are achieved by VOSA when the parameter $\operatorname{Vgfb}<10-15$. Vfgb is defined as the modified reduced $\chi^{2}$ and it is calculated by forcing $\sigma\left(F_{o b s}\right)>0.1 \times F_{o b s}$, where $\sigma\left(F_{o b s}\right)$ is the error of $F_{o b s}$ (the observed flux).

The SED was compared to three different grids of synthetic spectra, namely BT-Settl, BT-NextGen (AGSS2009) and BT-NextGen (GNS93) ${ }^{21}$. We note that we did not use other available libraries within VOSA due to the lack of models at low metallicites. During the fitting we accounted for interstellar extinction ${ }^{22}$ and used the Bayesian distance ${ }^{23}$ derived from the measured Gaia parallax and its uncertainty ${ }^{24}$ as the distance $\left(541_{-124}^{+216} \mathrm{pc}\right)$. We fixed the surface gravity to be within the range 5-5.5 dex, thus covering the 5.16 dex value that results from our mass and radius determinations from the light curve fit. Finally, the metallicity (i.e. Fe/H abundance) was assumed to be lower than $-1 \mathrm{dex}$, consistent with that of an usd star. In all cases $\mathrm{Vgfb}<15$, with our best-fit model achieving $\mathrm{Vgfb}=6.6$, which corroborates the goodness of our fit.

VOSA uses the bolometric flux and the distance to determine the luminosity. The bolometric flux is obtained integrating the flux using the observational photometric points. In cases where the SED is not covered by the observational photometry, VOSA uses the best-fit theoretical model.

\section{The iSpec $[\mathrm{Fe} / \mathrm{H}]$ determination}

The metallicity, in particular the $[\mathrm{Fe} / \mathrm{H}]$ abundance, of the cool subdwarf of SDSS J2355+0448 was additionally estimated using iSpec ${ }^{25}$, a code for the treatment and analysis of stellar spectra. iSpec allows determining the atmospheric stellar parameters (including the effective temperature, surface gravity, metallicity, micro- and macro-turbulence, rotation) and individual chemical abundances for FGKM stars via two different methods: (1) the synthetic spectral fitting technique and (2) the equivalent widths method. Here, we adopted the synthetic spectral fitting technique. This is implemented by iSpec via minimizing the $\chi^{2}$ between a given observed spectrum and an adopted library of synthetic spectra. We applied the synthetic spectral fitting technique to the observed (combined and white dwarf subtracted) X-Shooter spectrum of SDSS J2355+0448.

iSpec requires the user to mask the lines and corresponding segments to be used in the fitting. To that end we used the

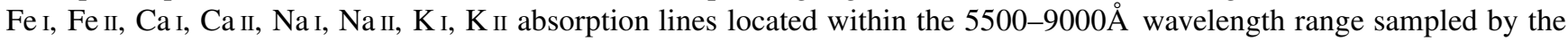
combined and white dwarf subtracted X-Shooter VIS arm spectrum. To do the computation of the $\chi^{2}$, iSpec used the GES (Gaia ESO survey) atomic line lists. The synthetic spectra were computed using the MARCS ${ }^{26}$ model atmosphere library 
and the SPECTRUM code within iSpec. An initial set of atmospheric parameters is also required prior to the fitting. We used $\mathrm{T}_{\text {eff }}=3650 \mathrm{~K}$ (based on our VOSA fitting results), $\log g=5.16 \mathrm{dex}$ (based on the mass and radius determined from the light curve fitting), $[\mathrm{M} / \mathrm{H}]=-1.55 \mathrm{dex}$ (we used the $[\mathrm{Fe} / \mathrm{H}]$ abundance derived from the $\tau_{\mathrm{TiO} / \mathrm{CaH}}=0.095 \mathrm{ratio}$ as a proxy for metallicity), $[\alpha / \mathrm{Fe}]=0$ dex, micro-turbulence velocity $V_{\text {mic }}=1.05 \mathrm{~km} \mathrm{~s}^{-1}$, macro-turbulence velocity $V_{\text {mac }}=4.21 \mathrm{~km} \mathrm{~s}{ }^{-1}$, $v \sin (i)=94.3 \mathrm{~km} \mathrm{~s}^{-1}$ (obtained from the measured orbital period of the binary and radius of the cool subdwarf, together with the known binary inclination), limb darkening coefficient $=0.6$, resolution $\mathrm{R}=8,900$ (set by our X-Shooter VIS spectrum) and radial velocity $=0 \mathrm{~km} \mathrm{~s}^{-1}$ (since the rest-frame radial velocity correction was performed before the fitting). The initial guesses of $\mathrm{T}_{\text {eff }}, \log g, v \sin (i), \mathrm{R}$ and radial velocity were fixed during the fitting process and the maximum number of iterations was set to 10 . This resulted in a value of $[\mathrm{Fe} / \mathrm{H}]=-2.1 \pm 2$ dex for the cool subdwarf star.

\section{The binary_c stellar evolution code}

We predict the future evolution of SDSS J2355+0448 by means of the binary stellar evolution code binary_c ${ }^{27,28}$, a software for the evolution of single and binary stars, calculations of stellar populations and nucleosynthesis analysis. binary_c updates and enhances the BSE (Binary Star Evolution) code ${ }^{29}$, providing stellar evolution for stars in the range 0.1 to $100 \mathrm{M}_{\odot}$. In order to simulate the evolution of a given binary star, binary_c requires a set of initial parameters such as the mass, stellar type (i.e. main sequence star, white dwarf, etc.), metallicity and rotational velocity of each star, as well as the orbital period, eccentricity and orbital separation of the binary. In all cases, except for the rotational velocity of the white dwarf and the metallicity of the stars, the input parameters are tightly constrained from our observations (note that the eccentricity must be zero for such a close binary star) and we hence used the observed values as input parameters in the simulation. We assumed a rotational velocity of $0 \mathrm{~km} \mathrm{~s}^{-1}$ for the white dwarf and we considered an $[\mathrm{Fe} / \mathrm{H}]$ abundance of -1.55 dex for the cool subdwarf. Additional parameters/assumptions are required by binary_c regarding explosions (not relevant in our case), Roche-lobe overflow, common envelope efficiency, winds and nucleosynthesis (also not relevant in our case). In those cases we assumed the standard values as provided by binary_c. We note however that modifying these assumptions had no dramatic effect in the evolutionary paths expected for SDSS J2355+0448, except slight changes in the evolutionary timescales.

\section{Code availability statement}

The spectral/decomposition routine and the radial-velocity fitting method used in this work are not publicly available. The

light-curve fitting method is available at https: / / github. com/trmrsh/cpp-l curve. MIDAS is available at https: / / www . esc VOSA is available at http://svo2. cab. inta-csic.es/theory/vosa/. iSpec is available at https: / / www. blancocuares The binary_c stellar evolution code is available at https://www.ast.cam.ac.uk/ rgi/binary_c.html. The XShooter reduction pipeline (version 2.9.3) is available at https : / www . eso.org/sci/software/pipelines/xshooter/ and the dedicated HiPERCAM pipeline at https: / / github.com/HiPERCAM/hipercam.

\section{Data availability statement}

Based on observations made with the Gran Telescopio Canarias (GTC), installed in the Spanish Observatorio del Roque de los Muchachos of the Instituto de Astrofísica de Canarias, in the island of La Palma (program GTC21-18B). Based on observations made with ESO Telescopes at the La Silla Paranal Observatory under programme ID 2100.D-5022(A).

This publication makes use of VOSA, developed under the Spanish Virtual Observatory project supported from the Spanish MINECO through grant AyA2017-84089. This work has made use of data from the European Space Agency (ESA) mission Gaia (https: / / www. cosmos.esa.int/gaia), processed by the Gaia Data Processing and Analysis Consortium (DPAC, https: / / www. cosmos.esa. int/web/gaia/dpac/consortium).

Figures 1, 3 and 4 and Supplementary Figures 1,2 and 4 have associated raw data. The data that support the plots within this paper and other findings of this study are available from the corresponding author upon reasonable request.

\section{References}

1. Vernet, J. et al. X-shooter, the new wide band intermediate resolution spectrograph at the ESO Very Large Telescope. Astron. Astrophys. 536, A105, DOI: 10.1051/0004-6361/201117752 (2011). 1110.1944.

2. Dhillon, V. et al. First light with HiPERCAM on the GTC. In Society of Photo-Optical Instrumentation Engineers (SPIE) Conference Series, vol. 10702 of Society of Photo-Optical Instrumentation Engineers (SPIE) Conference Series, 107020L, DOI: 10.1117/12.2312041 (2018). 1807.00557.

3. Rebassa-Mansergas, A., Gänsicke, B. T., Rodríguez-Gil, P., Schreiber, M. R. \& Koester, D. Post-common-envelope binaries from SDSS - I. 101 white dwarf main-sequence binaries with multiple Sloan Digital Sky Survey spectroscopy. Mon. Not. R. Astron. Soc. 382, 1377-1393, DOI: 10.1111/j.1365-2966.2007.12288.x (2007). 0707.4107. 
4. Rechenberg, I. Evolutionsstrategie '94 (Froomann-Holzboog, Stuttgart, 1994).

5. Rajpurohit, A. S. et al. Spectral energy distribution of M-subdwarfs: A study of their atmospheric properties. Astron. Astrophys. 596, A33, DOI: 10.1051/0004-6361/201526776 (2016). 1609.07062.

6. Kesseli, A. Y. et al. Radii of 88 M Subdwarfs and Updated Radius Relations for Low-Metallicity M Dwarf Stars. ArXiv e-prints (2018). 1810.07702.

7. Koester, D. White dwarf spectra and atmosphere models. Memorie della Soc. Astron. Italiana 81, 921-931 (2010).

8. Rebassa-Mansergas, A. et al. The white dwarf binary pathways survey - II. Radial velocities of 1453 FGK stars with white dwarf companions from LAMOST DR 4. Mon. Not. R. Astron. Soc. 472, 4193-4203, DOI: 10.1093/mnras/stx2259 (2017). 1708.09480.

9. Scargle, J. D. Studies in astronomical time series analysis. ii - statistical aspects of spectral analysis of unevenly spaced data. Astrophys. J. 263, 835-853 (1982).

10. Schwarzenberg-Czerny, A. Fast and statistically optimal period search in uneven sampled observations. Astrophys. J. 460, L107-L110 (1996).

11. Copperwheat, C. M. et al. Physical properties of IP Pegasi: an eclipsing dwarf nova with an unusually cool white dwarf. Mon. Not. R. Astron. Soc. 402, 1824-1840, DOI: 10.1111/j.1365-2966.2009.16010.x (2010). 0911.1637.

12. Bloemen, S. et al. Kepler observations of the beaming binary KPD 1946+4340. Mon. Not. R. Astron. Soc. 410, 1787-1796, DOI: 10.1111/j.1365-2966.2010.17559.x (2011). 1010.2747.

13. Claret, A. Non-linear limb-darkening law for LTE models (Claret, 2000). VizieR Online Data Catalog 336, 31081 (2000).

14. Gianninas, A., Strickland, B. D., Kilic, M. \& Bergeron, P. Limb-darkening Coefficients for Eclipsing White Dwarfs. Astrophys. J. 766, 3, DOI: 10.1088/0004-637X/766/1/3 (2013). 1301.7091.

15. Claret, A. \& Bloemen, S. Gravity and limb-darkening coefficients for the Kepler, CoRoT, Spitzer, uvby, UBVRIJHK, and Sloan photometric systems. Astron. Astrophys. 529, A75, DOI: 10.1051/0004-6361/201116451 (2011).

16. Parsons, S. G. et al. Testing the white dwarf mass-radius relationship with eclipsing binaries. Mon. Not. R. Astron. Soc. 470, 4473-4492, DOI: 10.1093/mnras/stx1522 (2017). 1706.05016.

17. Panei, J. A., Althaus, L. G., Chen, X. \& Han, Z. Full evolution of low-mass white dwarfs with helium and oxygen cores. Mon. Not. R. Astron. Soc. 382, 779-792, DOI: 10.1111/j.1365-2966.2007.12400.x (2007).

18. Althaus, L. G., De Gerónimo, F., Córsico, A., Torres, S. \& García-Berro, E. The evolution of white dwarfs resulting from helium-enhanced, low-metallicity progenitor stars. Astron. Astrophys. 597, A67, DOI: 10.1051/0004-6361/201629909 (2017). 1611.06191.

19. Press, W. H., Teukolsky, A. A., Vetterling, W. T. \& Flannery, B. P. Numerical recipes. The art of scientific computing, 3rd edn. (Cambridge: University Press, 2007).

20. Parsons, S. G. et al. The scatter of the M dwarf mass-radius relationship. Mon. Not. R. Astron. Soc. 481, 1083-1096, DOI: 10.1093/mnras/sty2345 (2018). 1808.07780.

21. Allard, F., Homeier, D. \& Freytag, B. Models of very-low-mass stars, brown dwarfs and exoplanets. Philos. Transactions Royal Soc. Lond. Ser. A 370, 2765-2777, DOI: 10.1098/rsta.2011.0269 (2012). 1112.3591.

22. Schlafly, E. F. \& Finkbeiner, D. P. Measuring Reddening with Sloan Digital Sky Survey Stellar Spectra and Recalibrating SFD. Astrophys. J. 737, 103, DOI: 10.1088/0004-637X/737/2/103 (2011). 1012.4804.

23. Bailer-Jones, C. A. L., Rybizki, J., Fouesneau, M., Mantelet, G. \& Andrae, R. Estimating Distance from Parallaxes. IV. Distances to 1.33 Billion Stars in Gaia Data Release 2. Astron. J. 156, 58, DOI: 10.3847/1538-3881/aacb21 (2018). 1804.10121.

24. Gaia Collaboration et al. Gaia Data Release 2. Summary of the contents and survey properties. Astron. Astrophys. 616, A1, DOI: 10.1051/0004-6361/201833051 (2018). 1804.09365.

25. Blanco-Cuaresma, S., Soubiran, C., Heiter, U. \& Jofré, P. Determining stellar atmospheric parameters and chemical abundances of FGK stars with iSpec. Astron. Astrophys. 569, A111, DOI: 10.1051/0004-6361/201423945 (2014). 1407.2608.

26. Gustafsson, B. et al. A grid of MARCS model atmospheres for late-type stars. I. Methods and general properties. Astron. Astrophys. 486, 951-970, DOI: 10.1051/0004-6361:200809724 (2008). 0805.0554.

27. Izzard, R. G., Tout, C. A., Karakas, A. I. \& Pols, O. R. A new synthetic model for asymptotic giant branch stars. Mon. Not. R. Astron. Soc. 350, 407-426, DOI: 10.1111/j.1365-2966.2004.07446.x (2004). astro-ph/0402403. 
28. Izzard, R. G. et al. Binary stars in the Galactic thick disc. Mon. Not. R. Astron. Soc. 473, 2984-2999, DOI: 10.1093/mnras/stx2355 (2018). 1709.05237.

29. Hurley, J. R., Tout, C. A. \& Pols, O. R. Evolution of binary stars and the effect of tides on binary populations. Mon. Not. R. Astron. Soc. 329, 897-928, DOI: 10.1046/j.1365-8711.2002.05038.x (2002). astro-ph/0201220.

\section{Acknowledgements}

This work was partially supported by the MINECO Ramón y Cajal programme RYJ-2016-20254 (A.R.M.) and grant AYA2017-86274-P(A.R.M., S.T.) and by the the AGAUR grant SGR-661/2017 (A.R.M., S.T.). S.G.P. acknowledges the support of the Leverhulme Trust. J.J.R. acknowledges support from the Joint Funds of the National Natural Sciences Foundation of China (Grant Nos U1531244 and U1831209), the NSFC grant 11833006 and the Young Researcher Grant of National Astronomical Observatories, Chinese Academy of Sciences. HiPERCAM and V.S.D are funded by the European Research Council under the European Union's Seventh Framework Programme (FP/2007-2013) under ERC-2013-ADG Grant Agreement no. 340040 (HiPERCAM).

The authors thank Fran Jimémez-Esteban for valuable input in the use of VOSA, Aurora Kesseli for sharing her usd spectra and Leandro Althaus for helpful discussions.

\section{Author contributions statement}

All authors have contributed to the work presented in this paper. A.R.M. performed the decomposition and fitting of the spectra, carried out the entire spectral analysis (except the one required by iSpec), conducted the VOSA analysis and led the writing of the manuscript. S.G.P. reduced all the spectroscopic and photometric data and carried out the light-curve analysis. V.S.D. and S.P.L. performed the GTC observations. J.J.R. conducted the iSpec analysis. V.S.D., S.P.L. and T.R.M. contributed to the development of HiPERCAM, a vital instrument for obtaining the results of this work. S.T. carried out the binary_c simulation and calculated the cooling age of the white dwarf. A.R.M., S.G.P. and J.J.R. discovered the system. All authors reviewed the manuscript.

\section{Additional information}

Competing Interests. The authors declare that they have no competing financial interests.

\begin{tabular}{lcccc}
\hline Parameter & $g_{s}$ & $r_{s}$ & $i_{s}$ & $z_{s}$ \\
\hline $\mathrm{WD} a_{1}$ & 0.6882 & 0.6126 & 0.5782 & 0.5530 \\
$\mathrm{WD} a_{2}$ & 0.1635 & 0.0232 & -0.1761 & -0.3300 \\
$\mathrm{WD} a_{3}$ & -0.4165 & -0.1818 & 0.0999 & 0.3024 \\
$\mathrm{WD} a_{4}$ & 0.1915 & 0.0823 & -0.0386 & -0.1209 \\
$\operatorname{sd} a_{1}$ & -0.4633 & -0.6869 & -0.3228 & -0.1407 \\
$\operatorname{sd} a_{2}$ & 2.3583 & 3.9532 & 2.9078 & 2.2760 \\
$\operatorname{sd} a_{3}$ & -1.4110 & -3.7931 & -2.8289 & -2.2345 \\
sd $a_{4}$ & 0.1903 & 1.1713 & 0.8566 & 0.6676 \\
sd grav. darkening & 1.1211 & 0.7539 & 0.6513 & 0.5731 \\
\hline
\end{tabular}

Table 2. Limb- and gravity-darkening coefficients used during our light curve fitting for the $g_{s}, r_{s}, i_{s}$ and $z_{s}$ bands. The white dwarf limb-darkening coefficients (WD $a_{1}$ to $\mathrm{WD} a_{4}$ ) are for a $\mathrm{T}_{\mathrm{eff}}=13,250 \mathrm{~K}, \log g=7.75 \mathrm{dex}$ white dwarf. The cool subdwarf limb-darkening (sd $a_{1}$ to sd $a_{4}$ ) and surface gravity (sd grav. darkening) coefficients are for a $\mathrm{T}_{\text {eff }}=3,650 \mathrm{~K}$, $\log g=5.0 \mathrm{dex},[\mathrm{Fe} / \mathrm{H}]=-2.0$ dex star. 

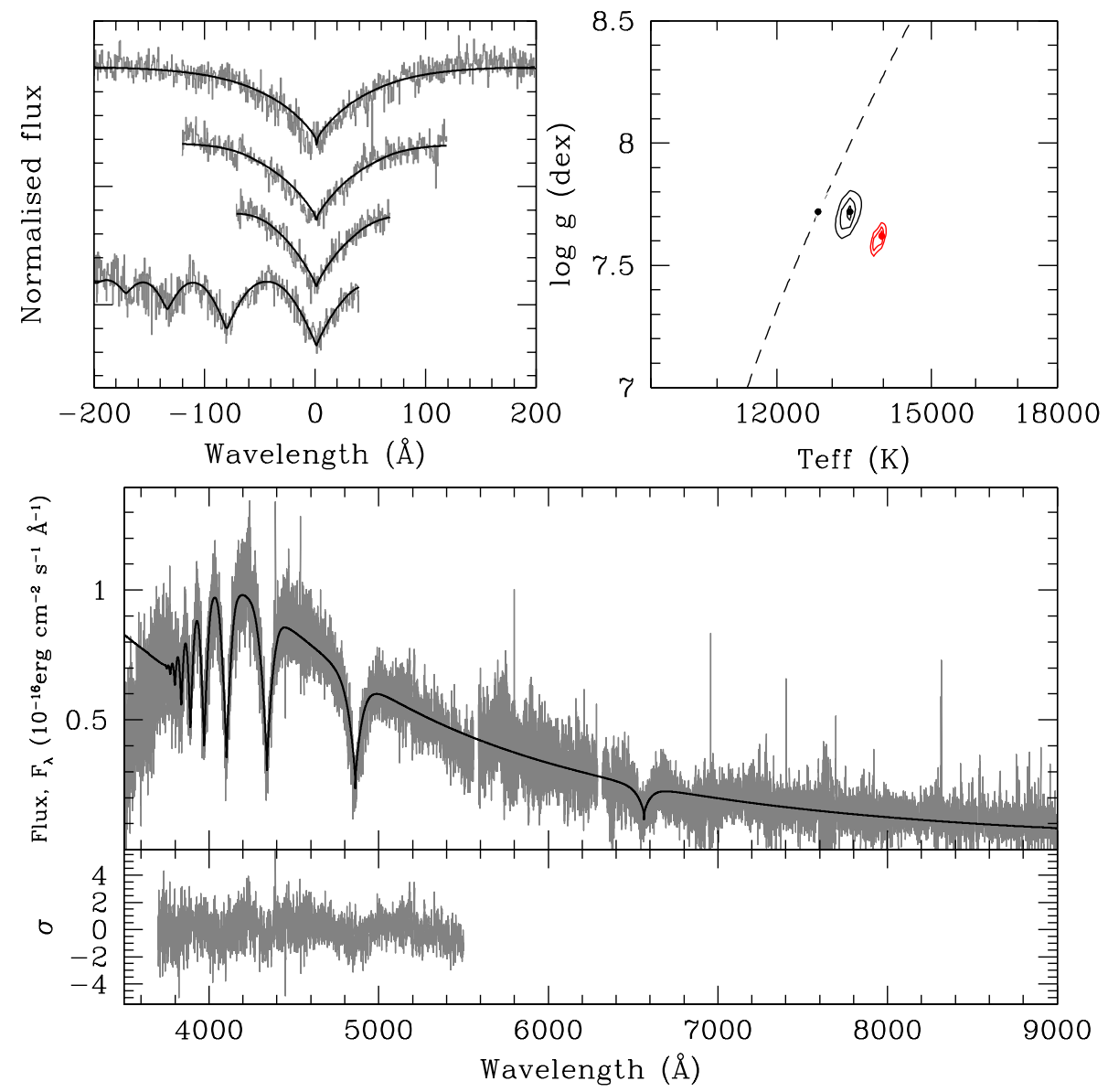

Figure 6. Spectral model fits to the residual white dwarf spectrum of SDSS J2355+0448, obtained after subtracting the best-fit cool subdwarf template (Figure 1). Top left panel: from top to bottom the best-fit (black line) to the normalised $\mathrm{H} \beta$ to $\mathrm{H} 10$ (gray) line profiles. Top right panel: 3,5 , and $10 \sigma \chi^{2}$ contour plots in the $\mathrm{T}_{\text {eff }}-\log g$ plane. Black contours indicate the best line profile fits, whilst red contours the best-fit to the entire spectrum. The maximum $\mathrm{H} \beta$ equivalent width is indicated by a dashed line. Black dots indicate the best "hot" and "cold" line profile solutions, the red dot indicates the best fit to the whole spectrum. Bottom panel: the residual white dwarf spectrum after subtracting the cool subdwarf (gray line) together with the best-fit white dwarf model (black line) and the residuals (gray line, bottom). The fit to the whole spectrum selects the "hot" solution. 


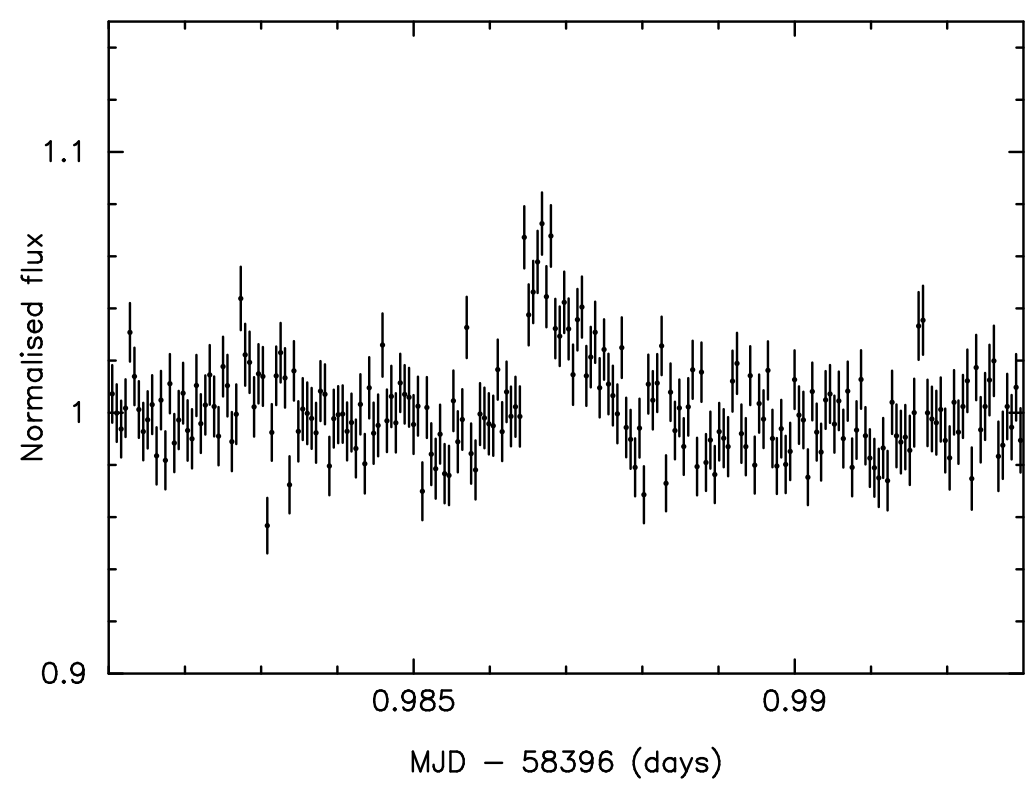

Figure 7. $g_{s}$-band HiPERCAM light-curve of SDSS J2355+0448 displaying a flare originating in the surface of the cool subdwarf star. The error bars represent $\pm 1 \sigma$ uncertainties.

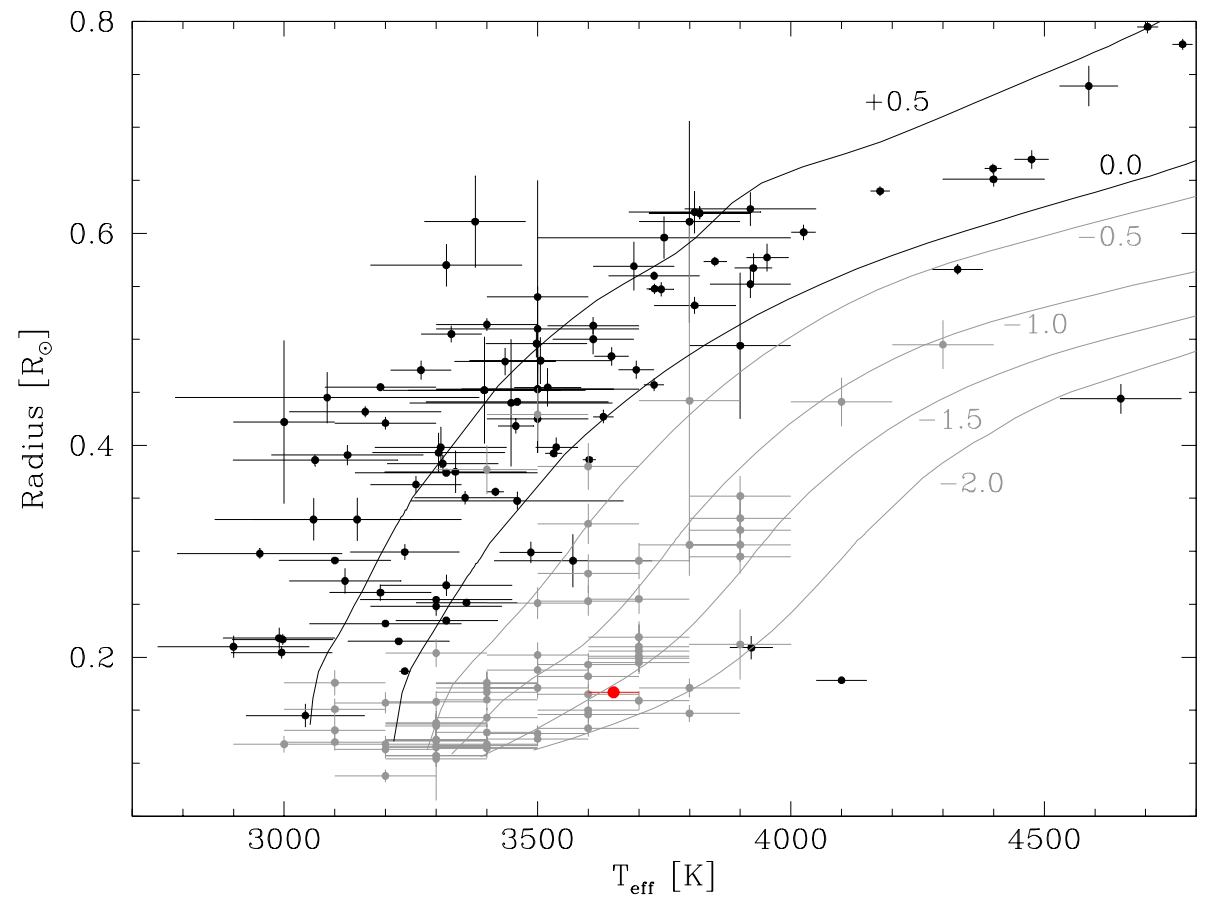

Figure 8. Radius-effective temperature plot for a compilation of low-mass solar-metallicity stars (black) and for a compilation of cool subdwarfs (grey). The solid lines are the Dartmouth theoretical tracks for $10 \mathrm{Gyr}$ and the indicated $[\mathrm{Fe} / \mathrm{H}]$ abundances (in dex units). The red solid dot represents the cool subdwarf studied in this work. The error bars represent $\pm 1 \sigma$ uncertainties. 


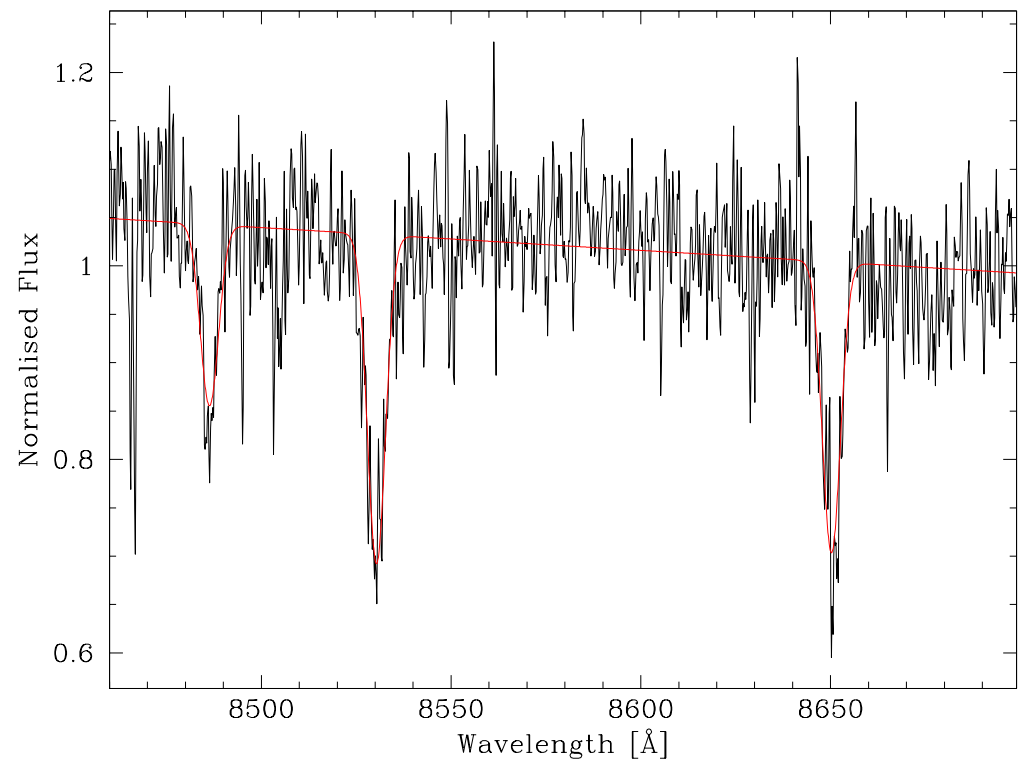

Figure 9. Fit (red solid line) to the normalised Ca II triplet at $\sim 8500 \AA ̊$ of a $\mathrm{X}$-Shooter spectrum (black solid lines). 